\title{
Existence and Multiplicity of Nontrivial Solutions for a Class of Semilinear Fractional Schrödinger Equations
}

\author{
Xinsheng Du and Anmin Mao \\ School of Mathematical Sciences, Qufu Normal University, Qufu, Shandong 273165, China \\ Correspondence should be addressed to Xinsheng Du; duxinsheng1977@163.com
}

Received 11 May 2017; Accepted 21 August 2017; Published 26 September 2017

Academic Editor: Yong H. Wu

Copyright (C) 2017 Xinsheng Du and Anmin Mao. This is an open access article distributed under the Creative Commons Attribution License, which permits unrestricted use, distribution, and reproduction in any medium, provided the original work is properly cited.

\begin{abstract}
This paper is concerned with the existence of solutions to the following fractional Schrödinger type equations: $(-\Delta)^{s} u+V(x) u=$ $f(x, u), x \in R^{N}$, where the primitive of the nonlinearity $f$ is of superquadratic growth near infinity in $u$ and the potential $V$ is allowed to be sign-changing. By using variant Fountain theorems, a sufficient condition is obtained for the existence of infinitely many nontrivial high energy solutions.
\end{abstract}

\section{Introduction and the Main Result}

In this work, under the assumptions that $V$ satisfies some weaker conditions than those in [1] and the primitive of $f$ satisfies a more general superquadratic condition near infinity, we study the existence of infinitely many nontrivial high energy solutions to the following fractional Schrödinger equations:

$$
(-\triangle)^{s} u+V(x) u=f(x, u), \quad x \in R^{N}
$$

where $s \in(0,1), N>2$, and $f: R^{N} \times R \rightarrow R$ is a continuous function with some proper growth conditions. Here $(-\triangle)^{s}$ is the so-called fractional Laplacian operator of order $s \in(0,1)$, which can be characterized as $(-\triangle)^{s} u=\mathscr{F}^{-1}\left(|\xi|^{2 s} \mathscr{F} u\right)$, with $\mathscr{F}$ being the usual Fourier transform in $R^{N}\left((-\triangle)^{s}\right.$ is the pseudodifferential operator with symbol $\left.|\xi|^{s}\right)$.

We need the following assumptions on $V(x)$ :

$\left(V_{1}\right) V \in C\left(R^{N}, R\right)$ is bounded from below.

$\left(V_{2}\right)$ For any $M>0$, there exists $r_{0}>0$ such that

$$
\lim _{|y| \rightarrow \infty} \operatorname{meas}\left(\left\{x \in R^{N}:|x-y| \leq r_{0}, V(x) \leq M\right\}\right)=0,
$$

where meas denotes the Lebesgue measure in $R^{N}$.
More recently, Di Nezza et al. [2] have proved that $(-\triangle)^{s}$ can be reduced to the standard Laplacian $-\Delta$ as $s \rightarrow 1$. When $s=1$, problem (1) is the classical Schrödinger equations

$$
(-\triangle) u+V(x) u=f(x, u), \quad x \in R^{N} .
$$

Over the past several decades, the existence and multiplicity of nontrivial solutions to problem (3) have been studied extensively by numerous researches using a variety of methods and techniques; see, for instance, [3-7].

The nonlinear equations involving fractional Laplacian, which is a powerful tool for the descriptions of physics, probability, and finance, have attracted the attention of many researchers and have been successfully applied in various fields; see, for instance, $[1,8,9]$ and the reference therein.

We need the following conditions on $f$ and its primitive $F(x, u)=\int_{0}^{u} f(x, s) d s:$

$\left(f_{1}\right) f \in C\left(R^{N} \times R, R\right)$ and there exist constant $C>0$ and $v \in\left(2,2_{s}^{*}\right)$ such that

$$
|f(x, u)| \leq C\left(|u|+|u|^{\nu-1}\right), \quad \forall(x, u) \in R^{N} \times R,
$$

where $2_{s}^{*}$ denotes the critical Sobolev exponent, that is, $2_{s}^{*}=$ $2 N /(N-2 s)$.

$\left(f_{2}\right) F(x, 0) \equiv 0, F(x, u) \geq 0$ for all $(x, u) \in R^{N} \times R$, and $\lim _{|u| \rightarrow \infty}\left(F(x, u) / u^{2}\right)=+\infty$ uniformly on $R^{N}$. 
$\left(f_{3}\right)$ There exists a constant $\theta \geq 1$ such that

$\theta \widetilde{F}(x, u) \geq \widetilde{F}(x, s u), \quad \forall(x, u) \in R^{N} \times R, s \in[0,1]$,

where $\widetilde{F}(x, u):=u f(x, u)-2 F(x, u)$.

$\left(f_{4}\right) f(x,-u)=-f(x, u), \forall(x, u) \in R^{N} \times R$.

Our main theorem of this work reads as follows.

Theorem 1. Suppose that $\left(V_{1}\right),\left(V_{2}\right)$, and $\left(f_{1}\right)-\left(f_{4}\right)$ hold. Then problem (1) possesses infinitely many nontrivial solutions.

Remark 2. Conditions like $\left(V_{1}\right)$ and $\left(V_{2}\right)$ have been given in [1], but there $\inf _{R^{N}} V(x)>0$ is required. As shown in [10], the condition $\left(f_{3}\right)$ due to [11] is somewhat weaker than the condition that $f(x, u) /|u|$ is nondecreasing in $u$ for all $x \in$ $R^{N}$.

Remark 3. It follows from $\left(V_{1}\right)$ that there exists a constant $V_{0} \geq 0$ such that $\bar{V}(x):=V(x)+V_{0} \geq 1$ for all $x \in R^{N}$. Let $\bar{f}(x, u)=f(x, u)+V_{0} u$ for all $(x, u) \in R^{N} \times R$ and consider the following new fractional Schrödinger equations

$$
(-\triangle)^{s} u+\bar{V}(x) u=\bar{f}(x, u), \quad x \in R^{N} .
$$

Then, problem (1) and problem (6) are equivalent. Evidently, the hypotheses $\left(V_{1}\right),\left(V_{2}\right)$, and $\left(f_{1}\right)-\left(f_{4}\right)$ still hold for $\bar{V}$ and $\bar{f}$ provided that those hold for $V$ and $f$. Hence, we can assume without loss of generality that $V(x) \geq 1$ for all $x \in R^{N}$ in $\left(V_{1}\right)$.

\section{The Proof of Main Result}

In this section, $s \in(0,1)$ is a fixed number. We denote by $\|\cdot\|_{p}$ the usual norm of the space $L^{P}\left(R^{N}\right) \cdot c_{i}(i=1,2, \ldots)$ or $C$ denotes some positive constants.

In the light of finite differences, the nonhomogeneous Sobolev space $H^{s}\left(R^{N}\right)$ is defined by

$$
\begin{aligned}
& H^{s}\left(R^{N}\right) \\
& \quad=\left\{u \in L^{2}\left(R^{N}\right): \frac{|u(x)-u(y)|}{|x-y|^{N / 2+s}} \in L^{2}\left(R^{N} \times R^{N}\right)\right\} .
\end{aligned}
$$

It is a Hilbert space, when endowed with the scalar product given by

$$
\begin{aligned}
& (u, v) \\
& =\int_{R^{N}} u v d x \\
& \quad+\int_{R^{N}} \int_{R^{N}} \frac{(u(x)-u(y))(v(x)-v(y))}{|x-y|^{N+2 s}} d x d y .
\end{aligned}
$$

The corresponding norm is therefore

$$
\begin{aligned}
& \|u\|_{H^{s}} \\
& =\left(\int_{R^{N}}|u|^{2} d x+\int_{R^{N}} \int_{R^{N}} \frac{|u(x)-u(y)|^{2}}{|x-y|^{N+2 s}} d x d y\right)^{1 / 2} .
\end{aligned}
$$

The space $H^{s}\left(R^{N}\right)$ is also denoted by the Fourier transform. Indeed, it is defined as follows:

$$
\begin{aligned}
& H^{s}\left(R^{N}\right)=\{u \\
& \left.\quad \in L^{2}\left(R^{N}\right): \int_{R^{N}}\left(1+|\xi|^{2}\right)^{s}|\mathscr{F} u(\xi)|^{2} d \xi<+\infty\right\} .
\end{aligned}
$$

This space has a Hilbert structure when endowed with the scalar product

$$
(u, v)=\int_{R^{N}}\left(1+|\xi|^{2}\right)^{s} \mathscr{F} u(\xi) \mathscr{F} v(\xi) d \xi
$$

so that the corresponding norm is

$$
\|u\|_{H^{s}}=\left(\int_{R^{N}}\left(1+|\xi|^{2}\right)^{s}|\mathscr{F} u(\xi)|^{2} d \xi\right)^{1 / 2}
$$

To illustrate the relationship of the above two norms, let us start from the concept of Schwartz function $\mathcal{S}$ (is dense in $H^{s}\left(R^{N}\right)$ ), that is, the rapidly decreasing $C^{\infty}$ function on $R^{N}$, which will be used later. If $u \in \mathcal{S}$, the fractional Laplacian $(-\triangle)^{s}$ acts on $u$ as

$$
\begin{aligned}
(-\triangle)^{s} u(x) & =C(N, s) \text { P.V. } \int_{R^{N}} \frac{u(x)-u(y)}{|x-y|^{N+2 s}} d y \\
& =C(N, s) \lim _{\epsilon \rightarrow 0^{+}} \int_{R^{N} \backslash B(0, \epsilon)} \frac{u(x)-u(y)}{|x-y|^{N+2 s}} d y,
\end{aligned}
$$

where the symbol P.V. represents the principle value of the integral and the constant $C(N, s)$ depends only on the space dimension $N$ and on the order $s$. We can write an integral expression for $C(N, s)$ in the form

$$
C(N, s)=\int_{R^{N}} \frac{1-\cos \zeta}{|\zeta|^{N+2 s}} d \zeta .
$$

In [2], the authors have proved

$$
\begin{aligned}
(-\Delta)^{s} u & =\mathscr{F}\left(|\xi|^{2 s} \mathscr{F} u\right), \\
{[u]_{H^{s}}^{2} } & =\frac{2}{C(N, s)} \int_{R^{N}}|\xi|^{2 s}|\mathscr{F} u|^{2} d \xi,
\end{aligned}
$$

where

$$
[u]_{H^{s}}=\left(\int_{R^{N}} \int_{R^{N}} \frac{|u(x)-u(y)|^{2}}{|x-y|^{N+2 s}} d x d y\right)^{1 / 2}
$$

is the Gagliardo (semi)norm. Moreover, by the Plancherel formula in Fourier analysis, it is easy to show that

$$
[u]_{H^{s}}^{2}=\frac{2}{C(N, s)}\left\|(-\Delta)^{s / 2} u\right\|_{L^{2}}^{2} .
$$

Hence, the norms on $H^{s}\left(R^{N}\right)$ which was defined above are all equivalent. 
For our problem (1), the Hilbert space $H$ is defined by

$$
\begin{aligned}
H & =\left\{u \in H^{s}\left(R^{N}\right): \int_{R^{N}}|\xi|^{2 s}|\mathscr{F} u|^{2} d \xi\right. \\
& \left.+\int_{R^{N}} V(x)|u|^{2} d x<+\infty\right\} .
\end{aligned}
$$

The inner product and the norm are defined as

$$
\begin{aligned}
(u, v) & =\int_{R^{N}} V(x) u v d x+\int_{R^{N}}|\xi|^{2 s} \mathscr{F} u(\xi) \mathscr{F} v(\xi) d \xi \\
\|u\| & =\left(\int_{R^{N}}|\xi|^{2 s}|\mathscr{F} u|^{2} d \xi+\int_{R^{N}} V(x)|u|^{2} d x\right)^{1 / 2} .
\end{aligned}
$$

Under the assumptions $\left(V_{1}\right)$ and $\left(V_{2}\right)$, we have the following lemma due to [10].

Lemma 4. The Hilbert space $H$ is compactly embedded in $L^{t}\left(R^{N}\right)$ for $2 \leq t<2_{s}^{*}=2 N /(N-2 s)$.

Definition 5. A weak solution to problem (1) is a function $u \in$ $H$ such that

$$
\begin{gathered}
\int_{R^{N}} V(x) u v d x+\int_{R^{N}}|\xi|^{2 s} \mathscr{F} u(\xi) \mathscr{F} v(\xi) d \xi \\
=\int_{R^{N}} f(x, u) v d x, \quad \forall u \in H .
\end{gathered}
$$

The energy functional associated with problem (1) is defined by

$$
\begin{aligned}
\Phi(u)= & \frac{1}{2} \int_{R^{N}}|\xi|^{2 s}|\mathscr{F} u(\xi)|^{2} d \xi+\frac{1}{2} \int_{R^{N}} V(x)|u|^{2} d x \\
& -\int_{R^{N}} F(x, u) d x, \quad u \in H .
\end{aligned}
$$

By Lemma 4 and conditions $\left(f_{1}\right)$ and $\left(f_{2}\right)$, we can prove that $\Phi$ is well defined and $\Phi \in C^{1}(H)$ with

$$
\begin{aligned}
\left\langle\Phi^{\prime}(u), v\right\rangle= & \int_{R^{N}} V(x) u v d x \\
& +\int_{R^{N}}|\xi|^{2 s} \mathscr{F} u(\xi) \mathscr{F} v(\xi) d \xi \\
& -\int_{R^{N}} f(x, u) v d x, \quad \forall u, v \in H .
\end{aligned}
$$

Therefore weak solutions of (1) correspond to critical points of $\Phi$.
Let $X$ be a Banach space with the norm $\|\cdot\|$ and let $\left\{X_{j}\right\}$ be a sequence of subspace of $X$ with $\operatorname{dim} X_{j}<+\infty$ for each $j \in \mathcal{N}$. Further, $X=\overline{\bigoplus_{j \in \mathcal{N}} X_{j}}$, the closure of the direct sum of all $X_{j}$. Set

$$
\begin{aligned}
& W_{k}=\bigoplus_{j=0}^{k} X_{j}, \\
& Z_{k}=\overline{\bigoplus_{j=k}^{\infty} X_{j},} \\
& B_{k}=\left\{u \in W_{k}:\|u\| \leq \rho_{k}\right\}, \\
& S_{k}=\left\{u \in Z_{k}:\|u\|=r_{k}\right\}
\end{aligned}
$$

for $\rho_{k}>r_{k}>0$. Consider a family of $C^{1}$ functionals $\Phi_{\lambda}$ : $X \rightarrow R$ defined by

$$
\Phi_{\lambda}(u)=A(u)-\lambda B(u), \quad \lambda \in[1,2] .
$$

The following variant Fountain theorem comes from Zou [12].

Theorem 6. Assume that the functional $\Phi_{\lambda}$ defined above satisfies the following:

$\left(A_{1}\right) \Phi_{\lambda}$ maps bounded sets to bounded sets uniformly for $\lambda \in[1,2]$, and $\Phi_{\lambda}(-u)=\Phi_{\lambda}(u)$ for all $(\lambda, u) \in[1,2] \times X$.

$\left(A_{2}\right) B(u) \geq 0$ for all $u \in X, A(u) \rightarrow+\infty$ or $B(u) \rightarrow+\infty$ as $\|u\| \rightarrow+\infty$.

$\left(A_{3}\right)$ There exists $\rho_{k}>r_{k}>0$ such that

$$
\begin{aligned}
b_{k}(\lambda) & =\inf _{u \in Z_{k},\|u\|=r_{k}} \Phi_{\lambda}(u)>a_{k}(\lambda) \\
& =\max _{u \in W_{k},\|u\|=\rho_{k}} \Phi_{\lambda}(u), \quad \forall \lambda \in[1,2] .
\end{aligned}
$$

Then

$$
b_{k}(\lambda) \leq c_{k}(\lambda)=\inf _{\gamma \in \Gamma_{k}} \max _{u \in B_{k}} \Phi_{\lambda}(\gamma(u)), \quad \forall \lambda \in[1,2],
$$

where $\Gamma_{k}=\left\{\gamma \in C\left(B_{k}, X\right): \gamma\right.$ is odd, $\left.\left.\gamma\right|_{\partial B_{k}}=i d\right\}$. Moreover, for almost every $\lambda \in[1,2]$, there exists a sequence $\left\{u_{n}^{k}(\lambda)\right\}$ such that

$$
\begin{aligned}
& \sup _{n}\left\|u_{n}^{k}(\lambda)\right\|<\infty, \\
& \Phi_{\lambda}^{\prime}\left(u_{n}^{k}(\lambda)\right) \longrightarrow 0, \\
& \Phi_{\lambda}\left(u_{n}^{k}(\lambda)\right) \longrightarrow c_{k}(\lambda)
\end{aligned}
$$


In order to use Theorem 6 to prove the main result, we define the functionals $A, B$, and $\Phi_{\lambda}$ on the working space $H$ by

$$
\begin{aligned}
A(u)= & \frac{1}{2} \int_{R^{N}}|\xi|^{2 s}|\mathscr{F} u(\xi)|^{2} d \xi \\
& +\frac{1}{2} \int_{R^{N}} V(x)|u|^{2} d x \\
B(u)= & \int_{R^{N}} F(x, u) d x \\
\Phi_{\lambda}(u)= & A(u)-\lambda B(u)=\frac{1}{2}\|u\|^{2}-\lambda \int_{R^{N}} F(x, u) d x
\end{aligned}
$$

for all $u \in H$ and $\lambda \in[1,2]$. We choose an orthonormal basis $\left\{e_{j}: j \in \mathcal{N}\right\}$ of $H$ and let $X_{j}=\operatorname{span}\left\{e_{j}\right\}$ for all $j \in \mathcal{N}$. Obviously $\Phi_{1}=\Phi$. In order to complete the proof of our theorem, we need the following lemmas.

Lemma 7. Assume that $\left(V_{1}\right),\left(V_{2}\right)$, and $\left(f_{1}\right)$ hold. Then there exists a positive integer $k_{1}$ and a sequence $r_{k} \rightarrow \infty$ as $k \rightarrow \infty$ such that

$$
b_{k}(\lambda):=\inf _{u \in Z_{k},\|u\|=r_{k}} \Phi_{\lambda}(u)>0, \quad \forall k \geq k_{1},
$$

where $Z_{k}=\overline{\bigoplus_{j=k}^{\infty} X_{j}}=\overline{\operatorname{span}\left\{e_{k}, \ldots\right\}}$ for all $k \in \mathcal{N}$.

Proof. From the definition of $\Phi_{\lambda}(u)$ and $\left(f_{1}\right)$, there holds

$$
\begin{aligned}
\Phi_{\lambda}(u) \geq & \frac{1}{2}\|u\|^{2}-2 \int_{R^{N}} F(x, u) d x \\
\geq \frac{1}{2}\|u\|^{2}-2 c_{1}\left(\|u\|_{2}^{2}+\|u\|_{v}^{v}\right), & \\
& \forall(\lambda, u) \in[1,2] \times H,
\end{aligned}
$$

where $c_{1}$ is a constant. Let

$$
\begin{aligned}
& l_{2}(k)=\sup _{u \in Z_{k},\|u\|=1}\|u\|_{2}, \\
& l_{v}(k)=\sup _{u \in Z_{k},\|u\|=1}\|u\|_{v},
\end{aligned}
$$

$\forall k \in \mathcal{N}$

Since $H$ is compactly embedded into both $L^{2}\left(R^{N}\right)$ and $L^{v}\left(R^{N}\right)$, we have

$$
\begin{aligned}
& l_{2}(k) \longrightarrow 0, \\
& l_{v}(k) \longrightarrow 0 \\
& \quad \text { as } k \longrightarrow \infty .
\end{aligned}
$$

From (30) and (31), it follows that

$$
\begin{array}{r}
\Phi_{\lambda}(u) \geq \frac{1}{2}\|u\|^{2}-2 c_{1} l_{2}^{2}(k)\|u\|^{2}-2 c_{1} l_{v}^{v}(k)\|u\|^{v}, \\
\forall(\lambda, u) \in[1,2] \times Z_{k} .
\end{array}
$$

From (32), there exists a positive integer $k_{1}$ such that

$$
2 c_{1} l_{2}^{2}(k) \leq \frac{1}{8}, \quad \forall k \geq k_{1}
$$

For each $k \geq k_{1}$, let $r_{k}=\left(16 c_{1} l_{v}^{v}(k)\right)^{1 /(2-v)}$. Then $r_{k} \rightarrow+\infty$ as $k \rightarrow \infty$, since $v>2$. It is immediate to check that

$$
b_{k}(\lambda):=\inf _{u \in Z_{k},\|u\|=r_{k}} \Phi_{\lambda}(u) \geq \frac{r_{k}^{2}}{4}>0, \quad \forall k \geq k_{1} .
$$

Hence, the proof of Lemma 7 is complete.

Lemma 8. Assume that $\left(V_{1}\right),\left(V_{2}\right),\left(f_{1}\right)$, and $\left(f_{2}\right)$ hold. Then for the positive integer $k_{1}$ and the sequence $\left\{r_{k}\right\}$ obtained in Lemma 7 , there exist $\rho_{k}>r_{k}$ for each $k \geq k_{1}$, such that

$$
a_{k}(\lambda)=\max _{u \in W_{k},\|u\|=\rho_{k}} \Phi_{\lambda}(u)<0, \quad \forall k \geq k_{1}
$$

where $W_{k}=\bigoplus_{j=1}^{k} X_{j}=\operatorname{span}\left\{e_{1}, \ldots, e_{k}\right\}$ for all $k \in \mathcal{N}$.

Proof. To begin with, we carry out that for any finite dimensional subspace $F \in H$ there exists a constant $\varepsilon>0$ such that

$$
\operatorname{meas}\left(\left\{x \in R^{N}:|u(x)| \geq \varepsilon\|u\|\right\}\right) \geq \varepsilon
$$

$\forall u \in F \backslash\{0\}$.

Assume for contradiction that, for any $n \in \mathcal{N}$, there exists $u_{n} \in F \backslash\{0\}$ such that

$$
\operatorname{meas}\left(\left\{x \in R^{N}:|u(x)| \geq \frac{\left\|u_{n}\right\|}{n}\right\}\right)<\frac{1}{n} .
$$

Let $v_{n}=u_{n} /\left\|u_{n}\right\| \in F$ for all $n \in \mathcal{N}$. Then $\left\|v_{n}\right\|=1$ for all $n \in \mathcal{N}$, and

$$
\operatorname{meas}\left(\left\{x \in R^{N}:\left|v_{n}(x)\right| \geq \frac{1}{n}\right\}\right)<\frac{1}{n}, \quad \forall n \in \mathcal{N} .
$$

Up to a subsequence, if necessary, we can say that $v_{n} \rightarrow v_{0}$ in $H$ for some $v_{0} \in F$ since $F$ is of finite dimension. Evidently, $\left\|v_{0}\right\|=1$. By Lemma 4 and the equivalence of any two norms on $F$, we conclude that

$$
\int_{R^{N}}\left|v_{n}-v_{0}\right|^{2} d x \longrightarrow 0 \quad \text { as } n \longrightarrow \infty
$$

Since $v_{0} \neq 0$, we see that there exists a constant $\delta_{0}>0$ satisfying

$$
\operatorname{meas}\left(\left\{x \in R^{N}:\left|v_{0}(x)\right| \geq \delta_{0}\right\}\right) \geq \delta_{0} .
$$

For any $n \in \mathcal{N}$, we define the sets

$$
\begin{aligned}
& \Lambda_{n}=\left\{x \in R^{N}:\left|v_{n}(x)\right| \leq \frac{1}{n}\right\}, \\
& \Lambda_{n}^{c}=R^{N} \backslash \Lambda_{n}=\left\{x \in R^{N}:\left|v_{n}(x)\right| \geq \frac{1}{n}\right\} .
\end{aligned}
$$


Let $\Lambda_{0}=\left\{x \in R^{N}:\left|v_{0}(x)\right| \geq \delta_{0}\right\}$. Then for sufficiently large $n$, from (39) and (41), one can easily see that

$$
\begin{aligned}
\operatorname{meas}\left(\Lambda_{n} \cap \Lambda_{0}\right) & \geq \operatorname{meas}\left(\Lambda_{0}\right)-\operatorname{meas}\left(\Lambda_{n}^{c}\right) \geq \delta_{0}-\frac{1}{n} \\
& \geq \frac{\delta_{0}}{2}
\end{aligned}
$$

So that, for $n$ large enough, we obtain

$$
\begin{aligned}
\int_{R^{N}}\left|v_{n}-v_{0}\right|^{2} d x & \geq \int_{\Lambda_{n} \cap \Lambda_{0}}\left|v_{n}-v_{0}\right|^{2} d x \\
& \geq \int_{\Lambda_{n} \cap \Lambda_{0}}\left(\left|v_{n}\right|-\left|v_{0}\right|\right)^{2} d x \\
& \geq\left(\delta_{0}-\frac{1}{n}\right)^{2} \cdot \operatorname{meas}\left(\Lambda_{n} \cap \Lambda_{0}\right) \\
& \geq \frac{\delta_{0}^{3}}{8}>0 .
\end{aligned}
$$

This is impossible, and (37) holds.

Since $W_{k}$ is finite dimensional for each $k \in \mathcal{N}$, we deduce from this and (37) that

$$
\operatorname{meas}\left(\Lambda_{u}^{k}\right) \geq \varepsilon_{k}, \quad \forall u \in W_{k} \backslash\{0\},
$$

where $\Lambda_{u}^{k}=\left\{x \in R^{N}:|u(x)| \geq \varepsilon_{k}\|u\|\right\}$ for all $k \in \mathcal{N}$ and $u \in W_{k} \backslash\{0\}$. By $\left(f_{2}\right)$, for each $k \in \mathcal{N}$, there exists a constant $b_{k}>0$ such that

$$
F(x, u) \geq \frac{u^{2}}{\varepsilon_{k}^{3}}, \quad \forall x \in R^{N},|u| \geq b_{k} .
$$

So, by (45), (46), and $\left(f_{2}\right)$, for any $k \in \mathcal{N}$ and $\lambda \in[1,2]$, we have

$$
\begin{aligned}
\Phi_{\lambda}(u) & \leq \frac{1}{2}\|u\|^{2}-\int_{R^{N}} F(x, u) d x \\
& \leq \frac{1}{2}\|u\|^{2}-\int_{\Lambda_{u}^{k}}\left(\frac{|u|^{2}}{\varepsilon_{k}^{3}}\right) d x \\
& \leq \frac{1}{2}\|u\|^{2}-\varepsilon_{k}^{2}\|u\|^{2} \cdot \frac{\operatorname{meas}\left(\Lambda_{u}^{k}\right)}{\varepsilon_{k}^{3}} \\
& \leq \frac{1}{2}\|u\|^{2}-\|u\|^{2}=-\frac{1}{2}\|u\|^{2}
\end{aligned}
$$

for all $u \in W_{k}$ with $\|u\| \geq b_{k} / \varepsilon_{k}$. Let

$$
\rho_{k}>\max \left\{r_{k}, \frac{b_{k}}{\varepsilon_{k}}\right\}, \quad \forall K \geq k_{1} .
$$

We see that

$$
a_{k}(\lambda):=a_{k}(\lambda)=\max _{u \in W_{k},\|u\|=\rho_{k}} \Phi_{\lambda}(u) \leq-\frac{\rho_{k}^{2}}{2}<0,
$$

$$
\forall k \geq k_{1} \text {. }
$$

The proof of Lemma 8 is complete.
Proof of Theorem 1. Now the assumption $\left(f_{1}\right)$ and the definition of $\Phi_{\lambda}(u)$ imply that $\Phi_{\lambda}$ maps bounded sets to bounded sets uniformly for $\lambda \in[1,2]$. From $\left(f_{4}\right)$, we obtain $\Phi_{\lambda}(u)=$ $\Phi_{\lambda}(-u)$ for all $(\lambda, u) \in[1,2] \times H$. Therefore, the condition $\left(A_{1}\right)$ of Theorem 6 holds. Clearly, the condition $\left(A_{2}\right)$ holds by the definitions of $A(u) B(u)$ and $\left(f_{2}\right)$. Moreover, Lemmas 7 and 8 imply that the condition $\left(A_{3}\right)$ holds for all $k \geq k_{1}$, where $k_{1}$ is given in Lemma 7. Thus, it follows from Theorem 6 that, for each $k \geq k_{1}$, a.e. $\lambda \in[1,2]$, there exists a sequence $\left\{u_{m}^{k}(\lambda)\right\} \subset H$ such that

$$
\begin{aligned}
\sup _{m}\left\|u_{m}^{k}(\lambda)\right\| & <\infty, \\
\Phi_{\lambda}^{\prime}\left(u_{m}^{k}(\lambda)\right) & \longrightarrow 0 \\
\Phi_{\lambda}\left(u_{m}^{k}(\lambda)\right) & \longrightarrow c_{k}(\lambda) \\
\text { as } m & \longrightarrow \infty,
\end{aligned}
$$

where

$$
c_{k}(\lambda)=\inf _{\gamma \in \Gamma_{k}} \max _{u \in B_{k}} \Phi_{\lambda}(\gamma(u)), \quad \forall \lambda \in[1,2]
$$

with $B_{k}=\left\{u \in W_{k}:\|u\| \leq \rho_{k}\right\}$ and $\Gamma_{k}=\left\{\gamma \in C\left(B_{k}, X\right)\right.$ : $\gamma$ is odd, $\left.\left.\gamma\right|_{\partial B_{k}}=i d\right\}$.

Furthermore, the proof of Lemma 7 shows that

$$
c_{k}(\lambda) \in\left[\overline{b_{k}}, \overline{c_{k}}\right], \quad \forall k \geq k_{1}, \lambda \in[1,2],
$$

where $\overline{c_{k}}=\max _{u \in B_{k}} \Phi_{1}(u)$ and $\overline{b_{k}}:=r_{k}^{2} / 4 \rightarrow \infty$ as $k \rightarrow \infty$.

For each $k \geq k_{1}$, (50) shows that we can choose $\lambda_{n} \rightarrow 1$ (depending on $k$ ) and can get the corresponding sequences satisfying

$$
\begin{aligned}
\sup _{m}\left\|u_{m}^{k}\left(\lambda_{n}\right)\right\|<\infty, & \\
\Phi_{\lambda_{n}}^{\prime}\left(u_{m}^{k}\left(\lambda_{n}\right)\right) \longrightarrow & 0 \\
& \text { as } m \longrightarrow \infty .
\end{aligned}
$$

We prove the following 2 claims.

Claim 1. For each $\lambda_{n}$ given above, the sequence $\left\{u_{m}^{k}\left(\lambda_{n}\right)\right\}_{m}^{\infty}$ has a convergent subsequence.

Throughout this paragraph, for the simplicity of notation, we denote $u_{m}=u_{m}^{k}\left(\lambda_{n}\right)$ by $m \in \mathcal{N}$ throughout this paragraph. Indeed, by (53), we may let

$$
u_{m} \rightarrow u_{0} \quad \text { as } m \longrightarrow \infty,
$$

for some $u_{0} \in H$. With the aid of Riesz Representation Theorem, $\Phi_{\lambda_{n}}^{\prime}: H \rightarrow H^{*}$ and $B^{\prime}: H \rightarrow H^{*}$ can be written as $\Phi_{\lambda_{n}}^{\prime}: H \rightarrow H$ and $B^{\prime}: H \rightarrow H$, respectively. Hence

$$
u_{m}=\Phi_{\lambda_{n}}^{\prime}\left(u_{m}\right)+\lambda_{n} B^{\prime}\left(u_{m}\right), \quad \forall m \in \mathcal{N}
$$


From Lemma 4, (53), (54), and (55), we see immediately that the right-hand side of (55) converges strongly in $H$, so that $u_{m} \rightarrow u_{0}$ in $H$, and then the proof of Claim 1 is complete.

Therefore, by Claim 1, up to subsequences, we may assume

$$
\lim _{m \rightarrow \infty} u_{m}^{k}\left(\lambda_{n}\right)=u_{n}^{k}, \quad \forall n \in \mathcal{N}, k \geq k_{1}
$$

and hence from (50) and (52) we deduce that

$$
\begin{aligned}
& \Phi_{\lambda_{n}}^{\prime}\left(u_{n}^{k}\right)=0, \\
& \Phi_{\lambda_{n}}\left(u_{n}^{k}\right) \in\left[\overline{b_{k}}, \overline{c_{k}}\right], \\
& \qquad n \in \mathcal{N}, k \geq k_{1} .
\end{aligned}
$$

Claim 2. For each $k \geq k_{1}$, the sequence $\left\{u_{n}^{k}\right\}_{n=1}^{\infty}$ in (56) is bounded.

Throughout this paragraph, for notational simplicity, we will write $u_{n}=u_{n}^{k}$ for all $n \in \mathcal{N}$.

Otherwise, up to subsequences, we may assume

$$
\begin{aligned}
\left\|u_{n}\right\| & \longrightarrow \infty, \\
w_{n} & :=\frac{u_{n}}{\left\|u_{n}\right\|} \rightarrow w_{0} \in H
\end{aligned}
$$

$$
\text { as } n \longrightarrow \infty \text {. }
$$

By Lemma 4 and (58), we can prove that

$$
w_{n} \longrightarrow w_{0} \text { in } L^{p}\left(R^{N}\right) \text { for } 2 \leq p<2_{s}^{*} .
$$

Up to subsequences, if necessary, we have

$$
w_{n}(x) \longrightarrow w_{0}(x) \quad \text { a.e. } x \in R^{N} \text {. }
$$

If $w_{0}=0$, then there exists a sequence $\left\{t_{n}\right\} \subset[0,1]$ such that

$$
\Phi_{\lambda_{n}}\left(t_{n} u_{n}\right)=\max _{t \in[0,1]} \Phi_{\lambda_{n}}\left(t u_{n}\right)
$$

For any $D>0$, setting $\overline{w_{n}}=\sqrt{4 D} w_{n}$, by (59) and $\left(f_{2}\right)$, we obtain that

$$
\begin{aligned}
0 & \leq \int_{R_{N}} F\left(x, \overline{w_{n}}\right) d x \leq C \int_{R^{N}}\left(\left|\overline{w_{n}}\right|^{2}+\left|\overline{w_{n}}\right|^{v}\right) d x \\
& \longrightarrow 0 \text { as } n \longrightarrow \infty .
\end{aligned}
$$

Thus, for $n$ large enough, there holds

$$
\begin{aligned}
\Phi_{\lambda_{n}}\left(t_{n} u_{n}\right) & \geq \Phi_{\lambda_{n}}\left(\overline{w_{n}}\right) \\
& =\frac{1}{2}\left\|\overline{w_{n}}\right\|^{2}-\lambda_{n} \int_{R^{N}} F\left(x, \overline{w_{n}}\right) d x \\
& =2 D-\lambda_{n} \int_{R^{N}} F\left(x, \overline{w_{n}}\right) d x \geq D .
\end{aligned}
$$

These yield immediately $\lim _{n \rightarrow \infty} \Phi_{\lambda_{n}}\left(t_{n} u_{n}\right)=+\infty$. Since $\Phi_{\lambda_{n}}(0)=0$ and $\Phi_{\lambda_{n}}\left(u_{n}\right) \in\left[\overline{b_{k}}, \overline{c_{k}}\right]$ in (57), we obtain $t_{n} \in(0,1)$ in (61) for $n$ large enough, which shows that

$$
0=\left.\frac{d}{d t}\right|_{t=t_{n}} \Phi_{\lambda_{n}}^{\prime}\left(t u_{n}\right)=\left\langle\Phi_{\lambda_{n}}^{\prime}\left(t u_{n}\right), t_{n} u_{n}\right\rangle .
$$

Hence, from (64) and $\left(f_{3}\right)$, we deduce that

$$
\begin{aligned}
\Phi_{\lambda_{n}}\left(u_{n}\right) & =\Phi_{\lambda_{n}}\left(u_{n}\right)-\frac{1}{2}\left\langle\Phi_{\lambda_{n}}^{\prime}\left(u_{n}\right), u_{n}\right\rangle \\
& =\frac{\lambda_{n}}{2} \int_{R^{N}} \widetilde{F}\left(x, u_{n}\right) d x \geq \frac{\lambda_{n}}{2 \theta} \widetilde{F}\left(x, t_{n} u_{n}\right) d x \\
& =\frac{1}{\theta} \Phi_{\lambda_{n}}\left(t_{n} u_{n}\right)-\frac{1}{2 \theta}\left\langle\Phi_{\lambda_{n}}^{\prime}\left(t_{n} u_{n}\right), t_{n} u_{n}\right\rangle \\
& =\frac{1}{\theta} \Phi_{\lambda_{n}}\left(t_{n} u_{n}\right) \longrightarrow+\infty \text { as } n \longrightarrow \infty,
\end{aligned}
$$

where $\theta$ is given in $\left(f_{3}\right)$. By (57), this is impossible.

Since $w_{0} \neq 0$, of course $\Omega:=\left\{x \in R^{N}: w_{0}(x) \neq 0\right\}$ has positive measure. Equation (58) shows that

$$
u_{n}(x) \longrightarrow \infty, \quad x \in \Omega
$$

From (58), (66), $\left(f_{2}\right)$, and Fatou's lemma, it is easy to see that

$$
\begin{aligned}
\frac{1}{2}-\frac{\Phi_{\lambda_{n}} u_{n}}{\left\|u_{n}\right\|^{2}} & =\lambda_{n} \int_{R^{N}} \frac{F\left(x, u_{n}\right)}{\left\|u_{n}\right\|^{2}} d x \\
& \geq \lambda_{n} \int_{\Omega}\left|w_{n}\right|^{2} \frac{F\left(x, u_{n}\right)}{\left|u_{n}\right|^{2}} d x \longrightarrow+\infty
\end{aligned}
$$

$$
\text { as } n \longrightarrow \infty \text {. }
$$

This contradiction shows that $w_{0} \neq 0$ cannot hold and concludes the proof of Claim 2.

For each $k \geq k_{1}$, using the same arguments in the proof of Claim 1, one can also prove that the sequence $\left\{u_{n}^{k}\right\}_{n=1}^{\infty}$ has a strong convergent subsequence with the limit $u^{k}$ being just the critical point of $\Phi=\Phi_{1}$ by Claim 2 and (57). Clearly, $\Phi\left(u^{k}\right) \in\left[\overline{b_{k}}, \overline{c_{k}}\right]$ for all $k \geq k_{1}$. These imply that $\Phi$ have infinitely many nontrivial critical points of since $\overline{b_{k}} \rightarrow+\infty$ as $k \rightarrow \infty$. Therefore, problem (1) admits infinitely many nontrivial solutions and the proof of Theorem 1 is complete.

\section{Conflicts of Interest}

The authors declare that there are no conflicts of interest regarding the publication of this paper.

\section{Acknowledgments}

This research is supported by the National Natural Science Foundation of China (11471187 and 11571197). 


\section{References}

[1] K. Teng, "Multiple solutions for a class of fractional Schrodinger equations in RN," Nonlinear Analysis: Real World Applications, vol. 21, pp. 76-86, 2015.

[2] E. Di Nezza, G. Palatucci, and E. Valdinoci, "Hitchhiker's guide to the fractional Sobolev spaces," Bulletin des Sciences Mathématiques, vol. 136, no. 5, pp. 521-573, 2012.

[3] Q. Zhang and B. Xu, "Multiplicity of solutions for a class of semilinear Schrodinger equations with sign-changing potential," Journal of Mathematical Analysis and Applications, vol. 377, no. 2, pp. 834-840, 2011.

[4] J. Sun, "Infinitely many solutions for a class of sublinear Schrodinger-Maxwell equations," Journal of Mathematical Analysis and Applications, vol. 390, no. 2, pp. 514-522, 2012.

[5] A. Mao, L. Yang, A. Qian, and S. Luan, "Existence and concentration of solutions of Schrödinger-Possion system," Applied Mathematics Letters, vol. 68, pp. 8-12, 2017.

[6] A. Mao and Z. Zhang, "Sign-changing and multiple solutions of Kirchhoff type problems without the P.S. condition," Nonlinear Analysis, vol. 70, no. 3, pp. 1275-1287, 2009.

[7] A. Mao and S. Luan, "Sign-changing solutions of a class of nonlocal quasilinear elliptic boundary value problems," Journal of Mathematical Analysis and Applications, vol. 383, no. 1, pp. 239-243, 2011.

[8] T. Bartsch, A. Pankov, and Z. Wang, "Nonlinear Schrodinger equations with steep potential well," Communications in Contemporary Mathematics, vol. 3, no. 4, pp. 549-569, 2001.

[9] L. Caffarelli and L. Silvestre, "An extension problem related to the fractional Laplacian," Communications in Partial Differential Equations, vol. 32, no. 7-9, pp. 1245-1260, 2007.

[10] S. B. Liu and S. J. Li, "Infinitely many solutions for a superlinear elliptic equation," Acta Mathematica Sinica, vol. 46, no. 4, pp. 625-630, 2003.

[11] L. Jeanjean, "On the existence of bounded Palais-smale sequence and application to a Landesman-Lazer type problem set on RN," Proceedings of the Royal Society of Edinburgh. Section A. Mathematics, vol. 129, no. 4, pp. 787-809, 1999.

[12] W. Zou, "Variant fountain theorems and their applications," Manuscripta Mathematica, vol. 104, no. 3, pp. 343-358, 2001. 


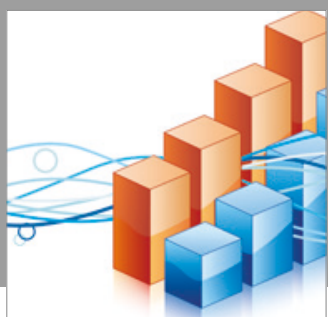

Advances in

Operations Research

vatersals

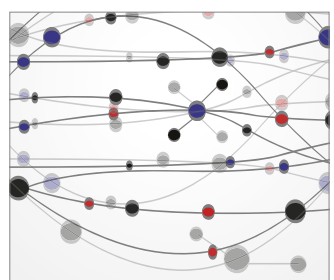

\section{The Scientific} World Journal
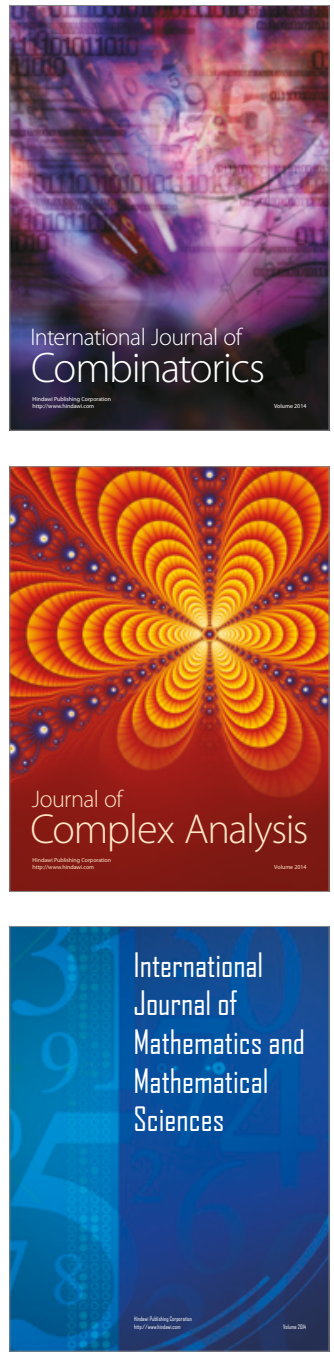
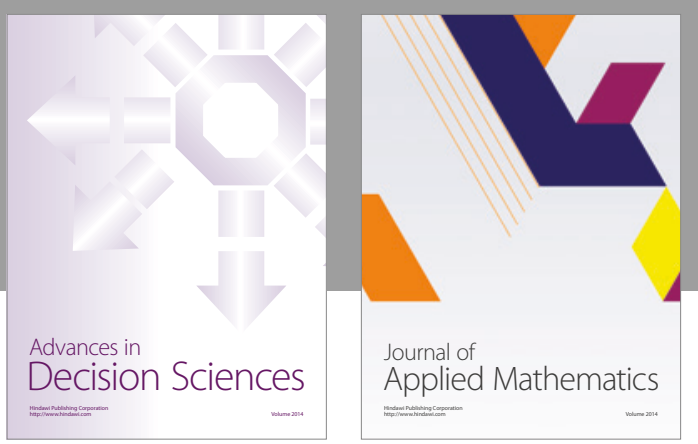

Algebra

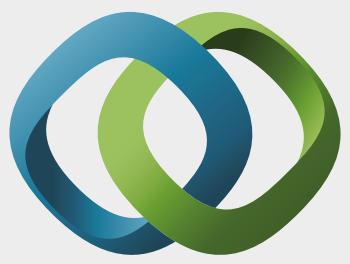

\section{Hindawi}

Submit your manuscripts at

https://www.hindawi.com
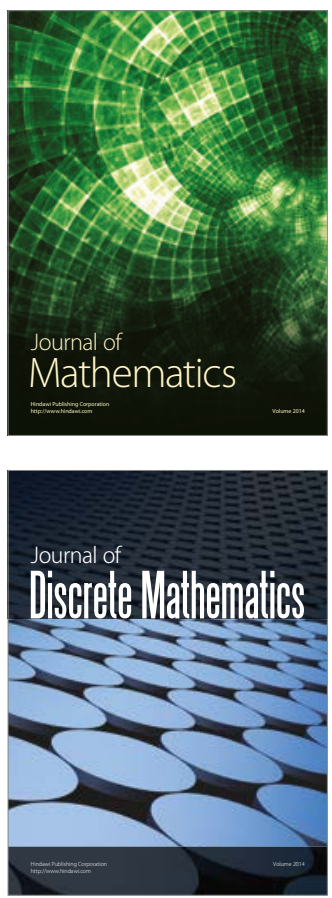

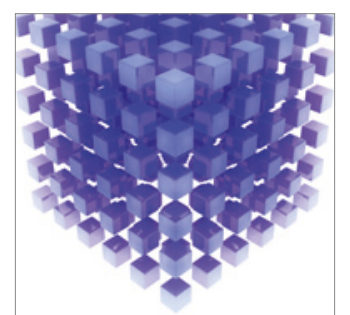

Mathematical Problems in Engineering
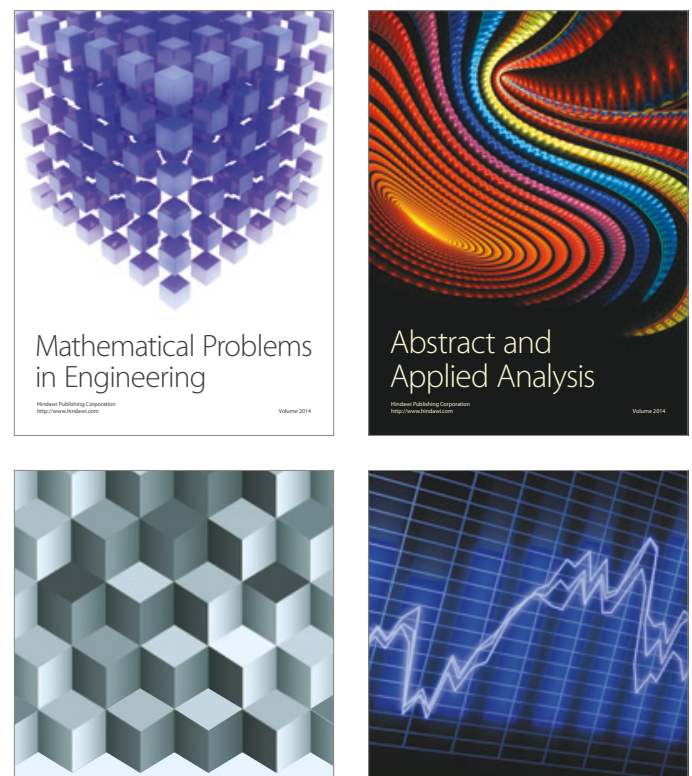

Journal of

Function Spaces

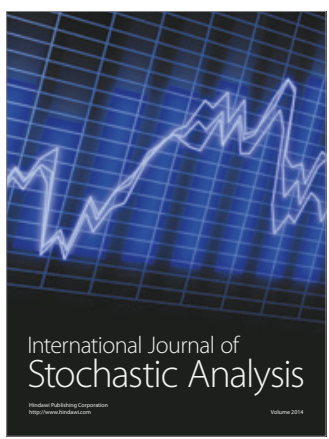

Probability and Statistics
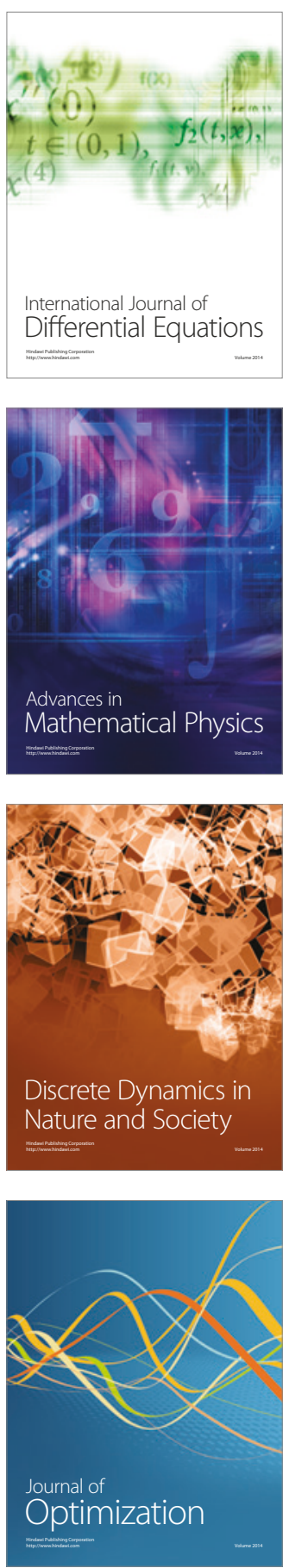